\title{
Hepatic miR-122 expression correlated with IL-28B genetic polymorphisms in hepatocellular carcinoma patients with living donor liver transplantation
}

\author{
Chih-Chi Wang ${ }^{\mathrm{a}, \mathrm{b}, \mathrm{c}}$, Kuang-Tzu Huang ${ }^{\mathrm{c}, \mathrm{d}}$, Kuang-Den Chen ${ }^{\mathrm{c}, \mathrm{d}}$, Li-Wen Hsu ${ }^{\mathrm{c}}$, Chih-Che Lin ${ }^{\mathrm{a}, \mathrm{b}, \mathrm{c}}$, \\ King-Wah Chiu ${ }^{\text {b,c,e,* }}$ \\ a Division of General Surgery, Department of Surgery, Kaohsiung Chang Gung Memorial Hospital, \\ Kaohsiung, Taiwan \\ b Chang Gung University, College of Medicine, Taoyuan, Taiwan \\ c Liver Transplantation Center, Department of Surgery, Kaohsiung Chang Gung Memorial Hospital, \\ Kaohsiung, Taiwan \\ ${ }^{\mathrm{d}}$ Institute for Translational Research in Biomedicine, Kaohsiung Chang Gung Memorial Hospital, \\ Kaohsiung, Taiwan \\ e Division of Hepato-Gastroenterology, Department of Internal Medicine, \\ Kaohsiung Chang Gung Memorial Hospital, Kaohsiung, Taiwan
}

*Corresponding author, e-mail: c471026@ms6.hinet.net

Received 2 Oct 2020

Accepted 25 Apr 2021

\begin{abstract}
Hepatocellular carcinoma (HCC) recurrence after living donor liver transplantation (LDLT) remains problematic. The genetic and molecular characteristics of patients may affect HCC recurrence. We evaluated the effects of microRNA-122 (miR-122) and interleukin-28B (IL-28B) genetic polymorphisms on patients with HCC following LDLT in 60 patients. MiR-122 and IL-28B polymorphisms were evaluated in plasma and liver tissues after LDLT. HBV, HCV, dual HBV/HCV infection, and non-B non-C were detected in 26, 22, 3, and 9 patients, respectively, over a median follow-up time of 20.5 (10-33) months. miR-122 was significantly higher in the liver than in the plasma of patients with HBV, HCV, dual HBV/HCV, and non-B non-C. Hepatic miR-122 expression was significantly higher for genotype TT and genotype TT plus GT ( $p=0.005)$ compared with genotype GG of IL-28B rs8099917 and significantly higher in $>6 \%$ fatty liver than in $<5 \%$ or no fatty liver. In conclusion, high hepatic miR-122 was correlated with the IL-28B rs8099917 genotypes TT and GT and with $>6 \%$ fatty liver and, thus, may play a major role in HCC.
\end{abstract}

KEYWORDS: fatty liver, hepatocellular carcinoma, interleukin-28B, living donor liver transplantation, microRNA-122

\section{INTRODUCTION}

MicroRNAs (miRs) are single-stranded small molecules that can regulate the expression levels of their target genes by binding to $3^{\prime}$-untranslated regions [1]. miR-122 can specifically bind to hepatitis B virus (HBV) RNA and, then, be downregulated in HBV-related hepatocellular carcinoma (HCC) [2]. Thus, miR-122 is a diagnostic and prognostic predictor of HCC $[3,4]$. Interleukin-28B (IL-28B) genetic polymorphisms rs8099917 and rs12979860 contribute to hepatitis $\mathrm{C}$ virus (HCV)-related HCC [5]. Although serum miR-122 may serve as a surrogate of hepatic miR-122 [6], overexpression of hepatic miR-301a induces interleukin- 6 production in rat primary hepatocytes [7]. Therefore, hepatic microRNA
(miRNA) expression should be evaluated to determine the relationship between IL genetic polymorphisms and HCC. Plasma and liver tissue expressions of miR-122 in the native liver were evaluated in this study. Additionally, serum IL28B with the rs8099917 and rs12979860 genetic polymorphisms was examined to determine its correlation with the pathologic characteristics of patients with HCC who underwent living donor liver transplantation (LDLT).

\section{PATIENTS AND METHODS}

\section{General information}

Between September 2016 and August 2018, 60 patients with HCC who had undergone LDLT were enrolled consecutively in this prospective cohort 
Table 1 Clinical profiles of the 60 patients, associated with hepatocellular carcinoma, who underwent living donor liver transplantation.

\begin{tabular}{ll}
\hline Category & Patients $(n=60)$ \\
\hline Age, mean \pm SD (range) (years) & $57.78 \pm 7.16(39-68)$ \\
Gender, male/female & $14 / 46$ \\
Anti-HCV (+) (\%) & $25(41.7)$ \\
Genotypes 1, 1a, 1b, 1b/2, 2, unknown & $1,1,8,1,6,8$ \\
HCV-RNA $>4 \times 10^{5}$ IU/ml, (\%) & $12(48.0)$ \\
HCV-RNA < $4 \times 10^{5}$ IU/ml, (\%) & $3(12.0)$ \\
HCV-RNA (-) (\%) & $10(40.0)$ \\
HBsAg (+) before LDLT (\%) & $29^{*}(48.3)$ \\
HBV-DNA >2000 IU/ml, (\%) & $4(13.8)$ \\
HBV-DNA <2000 IU/ml, (\%) & $6(20.7)$ \\
HBV-DNA (-) (\%) & $19(65.5)$ \\
HBeAg (+)/anti-HBe (+) & $1 / 28$ \\
HBsAg (+)/HBcAg (+) in native liver & $21 / 2$ \\
HBsAg (-), anti-HBs (+) after LDLT (\%) & $29(100.0)$ \\
Anti-HCV (-), HBsAg (-), anti-HBs $(-)$ & $9(15.0)$ \\
before LDLT (\%) & \\
AFP (ng/dl), mean \pm SD & $16.44 \pm 36.48$ \\
\hline
\end{tabular}

" Both positive for anti-HCV and HBsAg in 3 cases; AFP, serum alpha fetoprotein; LDLT, living donor liver transplantation; and SD, standard deviation.

study. Of the 60 patients, 46 were male and 14 were female with a mean age of 57.78 years (range, 39-68 years). The general demographic information and clinical characteristics; including hepatitis markers, viral load, anti-viral treatment and response, and postoperative pathological characteristics; are shown in Table 1. Twenty-six (43.3\%) patients showed a positive response to $\mathrm{HBsAg}, 22$ (36.7\%) showed a positive response to anti-HCV antibody, $3(5 \%)$ showed a positive response to both of HBsAg and anti-HCV antibodies, and 9 (15\%) showed a negative response to both of $\mathrm{HBsAg}$ and anti-HCV antibodies. Of these patients, 10 harbored HCV genotype 1, 7 showed HCV nongenotype 1 , and 8 showed uncertain genotypes because HCV-RNA levels were undetectable before LDLT. All patients with positive HCV-RNA were administered pre-transplant direct-acting antiviral agents for 3 months as Harvoni (sofosbuvir $400 \mathrm{mg}$ plus ledipasvir $90 \mathrm{mg}$ )/tab (one tab per day), or sofosbuvir $400 \mathrm{mg}$, dacltasvir $60 \mathrm{mg}$, ribavirin $800 \mathrm{mg}$ regimens, based on their HCV genotype. All patients were serum-negative for HCV-RNA before LDLT. For patients who were serum-positive for HBsAg, pretransplant nucleos(t)ide analogue with entecavir or tenofovir disoproxil fumarate were administered. Hepatitis B immunoglobulin therapy was administered in the anhepatic phase as a single intravenous dose of $10000 \mathrm{IU}$, which was continued after LDLT at $2170 \mathrm{IU} /$ day intramuscularly for 7 days, followed by 2170 IU intramuscularly as needed to maintain a trough anti-HBs antibody level of $>500 \mathrm{IU} / 1$ for 3 months, $>200 \mathrm{IU} / 1$ for 3-6 months, and $>$ $100 \mathrm{IU} / 1$ for $>6$ months. All patients who were serum-positive for HBsAg showed sero-conversion to negative results and $100 \%$ of patients developed anti-HBs antibody after LDLT. The mean serum AFP level was $16.44 \pm 36.48 \mathrm{ng} / \mathrm{dl}$ in our series. Inclusion criteria were a pathological diagnosis of HCC and adherence fit for Barcelona Clinic Liver Cancer (BCLC) system treatment guidelines. Exclusion criteria included pediatric liver transplantation, underlying psychological disease, and alcohol-related liver disease. Major vessel invasion was an absolute contraindication for LDLT.

\section{Real-time quantitative reverse transcription-PCR (RT-PCR) for plasma and liver tissue}

We evaluated miR-122 levels in the native liver tissue after LDLT. Quantitative RT-PCR was performed to validate the gene expression microarray data. Total RNA was isolated from the liver tissues, using the miRNeasy Mini Kit, and from the plasma, using the miRNeasy Serum/Plasma Kit from Qiagen (Hilden, Germany), according to the manufacturer's protocol. Reverse transcription (RT) was performed with $1 \mu \mathrm{g}$ RNA using the First-Strand cDNA Synthesis Kit (Promega, Madison, WI, USA) or miScript RT Kit (Qiagen) for the transcription of miRNA according to the manufacturer's instructions. Quantitative RT-PCR was performed on an ABI 7500 Fast RealTime PCR System with the SDS 1.4 program using ABI TaqMan Fast Universal PCR master mix or TaqMan Universal PCR master mix for miRNA (Applied Biosystems, Foster City, CA, USA). The primers and TaqMan MGB probes were obtained from Applied Biosystems, and the final concentrations of primers and probes were 300 and $250 \mathrm{nM}$, respectively. The cycling profile for each run was $95^{\circ} \mathrm{C}$ for $20 \mathrm{~s}$ and 40 cycles of $95^{\circ} \mathrm{C}$ for $3 \mathrm{~s}$ followed by $60^{\circ} \mathrm{C}$ for $30 \mathrm{~s}$ using the default ramp rate. The results were normalized to the levels of glyceraldehyde 3phosphate dehydrogenase. For miRNA, the primers and TaqMan probes for miR-122 (P/N: 4427975, ID: 002245) and U6 snRNA (P/N: 4427975, ID: 001973) were obtained from Applied Biosystems. The cycling profile of each run was $50^{\circ} \mathrm{C}$ for $2 \mathrm{~min}$, $95^{\circ} \mathrm{C}$ for $10 \mathrm{~min}$, and 40 cycles of $95^{\circ} \mathrm{C}$ for $15 \mathrm{~s}$, followed by $60^{\circ} \mathrm{C}$ for $1 \mathrm{~min}$ using the default ramp rate. The cycle threshold (Ct value) is defined as the number of cycles required for the fluorescent signal to cross the threshold in qPCR and is inversely correlated with the miRNA level. Normalization was performed with U6 small nuclear RNA primers. 
Comparative RT-PCR data including non-template controls were obtained in triplicate. The foldincrease in cytokine mRNA expression was calculated using the comparative $2^{-\Delta \Delta \mathrm{Ct}}$ method, where Ct represents the threshold cycle for each transcript. The expression of the miRNA was defined based on $\mathrm{Ct}$, and relative expression levels were calculated as $2^{-[(\mathrm{Ct} \text { of miR-122)-(Ct of U6)] }}$ after normalization with reference to the expression of small nuclear RNA U6 [8].

\section{IL-28B rs8099917 and rs12979860 polymorphisms}

Genomic DNA was extracted from the peripheral blood mononuclear cells of the patients on the day of operation using a QIAamp DNA Blood Mini Kit (Qiagen). Genotyping of IL-28B polymorphisms was performed using ABI Custom TaqMan SNP Genotyping Assays and an allelic discrimination kit (Applied Biosystems, Foster City, CA, USA). Realtime PCR was performed in 96-well microplates using the ABI 7500 Fast Real-Time Polymerase Chain Reaction System (Applied Biosystems International, Framingham, MA) in accordance with the manufacturer's instructions [5]. The IL28B SNP rs8099917 was defined as TT, GT, or GG genotypes; and rs12979860 was defined as CC, CT, or TT genotypes, as recommended by the manufacturer. All genotypes of $I L-28 B$ SNP rs8099917 and rs12989860 were assayed in duplicate to assess inter-assay precision; and the results are shown in Fig. 1.

\section{Ethics}

All procedures involving human participants were performed in accordance with the ethical standards of the institutional and/or national research committee and with the 1964 Helsinki Declaration and its later amendments or comparable ethical standards. The study was approved and authorized by the hospital's ethical committee (Chang Gung Memorial Hospital of Taiwan; ethical approval number: 201600468B0C502). An informed consent was obtained from all subjects included in the study. No transplant donors or patients were from a vulnerable population, and a written informed consent was provided by all donors or their next of kin.

\section{Statistics}

Descriptive values are expressed as the mean (SD) and percentages as appropriate. The $\chi^{2}$ test was used for categorical data. Spearman correlation was used to examine the relationship between serum
miR-122 and IL28B genetic polymorphisms. A nonparametric trend test was used for ordinal data. The p-values were two-tailed, and those $<0.05$ were considered to indicate statistical significance.

\section{RESULTS}

\section{MiR-122 in the plasma and the liver tissue}

miR-122 expression was significantly higher in the native liver tissue than in the plasma, as shown in Fig. 2, among the different etiologies including HBV $(p<0.05)$, HCV $(p<0.005)$, dual HBV/HCV $(p<0.05)$, and non-B non-C $(p<0.05)$ related (Table 2). The meaning of $p$-value showed that miR-122 expression was significantly higher in the liver tissue than those in the plasma as well as the subgroups.

\section{Correlation between hepatic miR-122 and IL-28B genetic polymorphism}

A total of $58.3 \%(35 / 60)$ patients displayed the rs8099917 genotype TT, 30.0\% (18/60) had genotype GT, and $11.7 \%(7 / 60)$ had genotype GG. Hepatic miR-122 expression was significantly higher in patients with genotype TT $(2.22 \pm 2.82)$ than in those with genotype GG $(0.85 \pm 0.58)(p<0.05)$, and higher hepatic miR-122 expression was observed in patients with genotype TT plus genotype GT $(2.80 \pm 5.73)$ than in those with genotype GG $(p<0.05)$ (Fig. 3).

There was no significant difference in hepatic miR-122 expression between patients with the rs12979860 genotype CT $(80.0 \%, 48 / 60)$ and TT $(20.0 \%, 12 / 60)(2.31 \pm 4.02$ vs. $1.91 \pm 2.86, p>$ $0.05)$.

\section{Hepatic miR-122 expression in histopathological characteristics}

Based on the classification of American Joint Committee on Cancer and BCLC System, 96.7\% (58/60) of patients were in HCC stages I and II. According to the Knodell score, $95.0 \%(57 / 60)$ of patients showed F4 fibrosis (Table 2). Examination of the association with the fatty component in the native liver revealed that $45 \%(27 / 60)$ of patients did not have fatty liver, $41.7 \%(25 / 60)$ had $<5 \%$ fatty liver, and $13.3 \%(8 / 60)$ had $>6 \%$ fatty liver (Table 2$)$. Hepatic miR-122 expression was significantly different between the native $>6 \%$ fatty liver and without fatty liver $(5.99 \pm 8.24$ vs. $1.68 \pm 2.52, p<0.005)$ groups, as well as between the $>6 \%$ and $<5 \%$ fatty liver groups ( $5.99 \pm 8.24$ vs. $1.63 \pm 1.82, p<0.005$ ) 

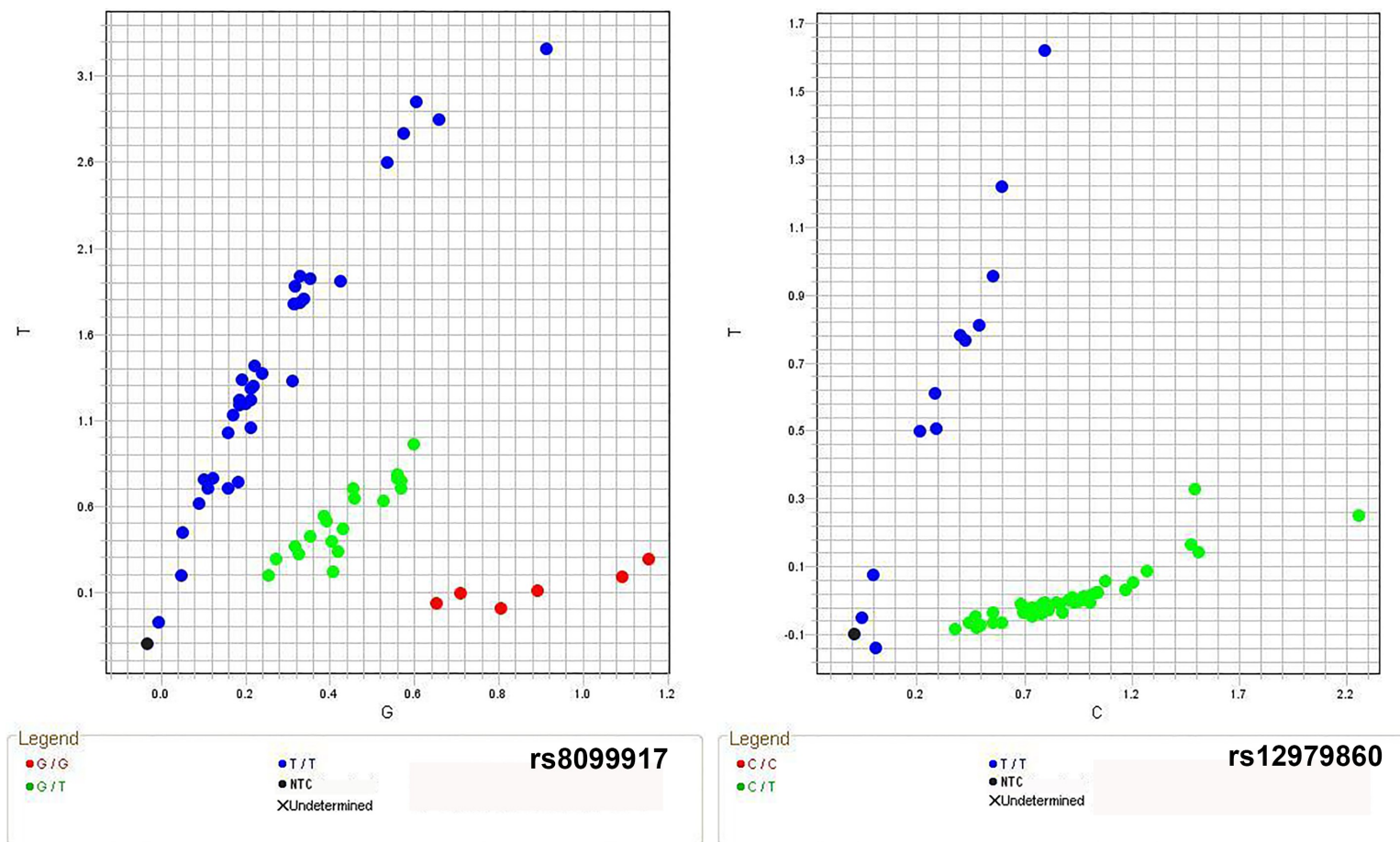

Fig. 1 Real-time polymerase chain reaction to evaluate IL28B rs8099917 polymorphisms genotypes GG, GT, and TT (left side) and rs12979860 genotypes TT, CT, and CC (right side) in the peripheral blood of patients on the day of living donor liver transplantation. Genotype CC in rs12979860 was not detected.
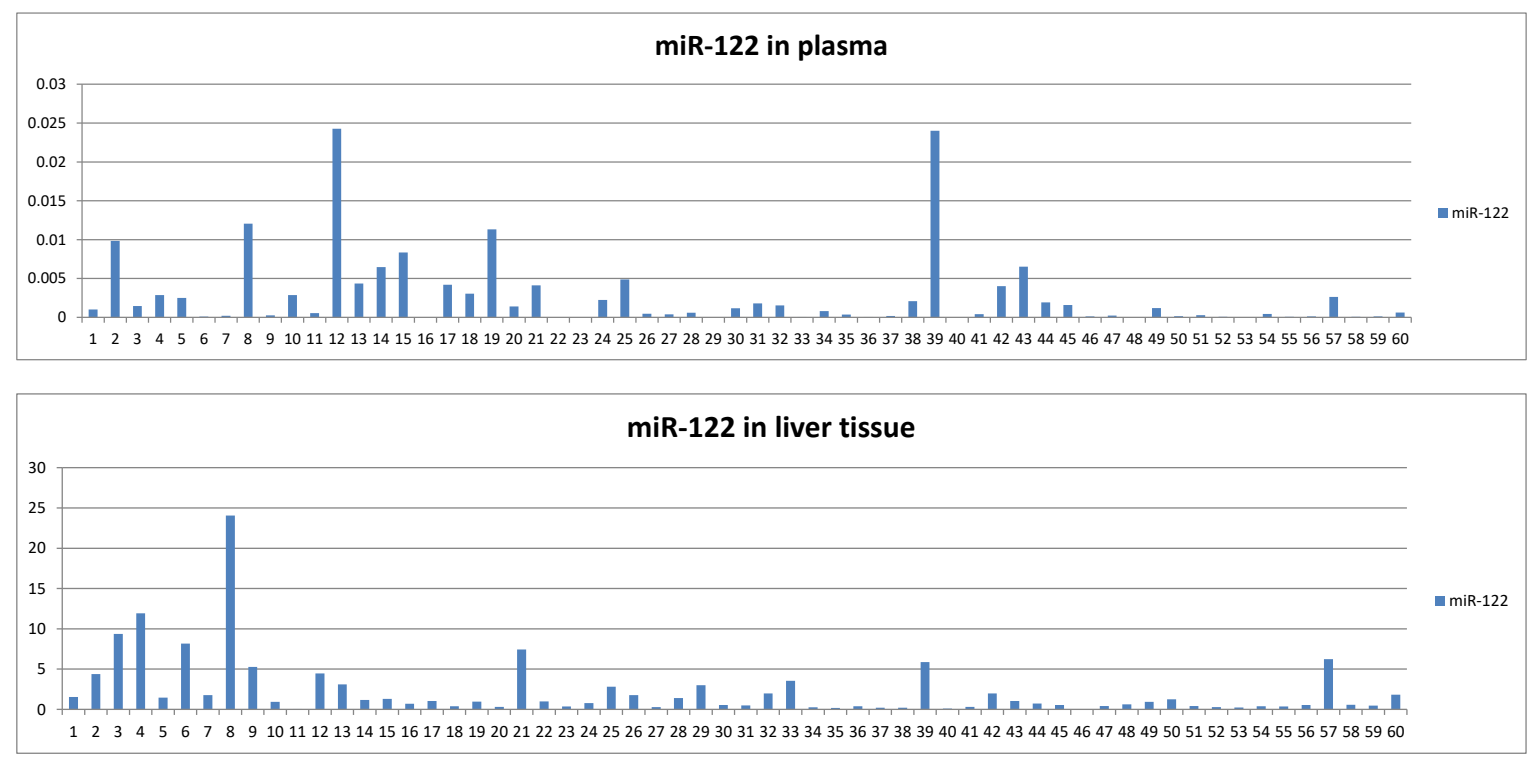

Fig. 2 MiR-122 in plasma/liver tissue of 60 patients associated with hepatocellular carinoma who underwent living donor liver transplantation. X-axis was defined as case numbers, and Y-axis was defined as cycle threshold (Ct value) of the miR-122 expression level. 
Table 2 MiR-122 level in the plasma and native liver of 60 patients, with different etiologies associated with hepatocellular carcinoma, who underwent living donor liver transplantation.

\begin{tabular}{|c|c|c|c|c|}
\hline Category & HBV, $\mathrm{n}=26(\%)$ & HCV, $\mathrm{n}=22(\%)$ & $\mathrm{HBV} / \mathrm{HCV}(+), \mathrm{n}=3(\%)$ & $\mathrm{HBV} / \mathrm{HCV}(-), \mathrm{n}=9(\%)$ \\
\hline miR-122 (liver), mean \pm SD & $2.93 \pm 5.15^{\mathrm{a}}$ & $1.49 \pm 2.07^{\mathrm{b}}$ & $0.63 \pm 0.21^{\mathrm{c}}$ & $2.58 \pm 2.82^{\mathrm{d}}$ \\
\hline miR-122 (plasma), mean \pm SD & $0.003 \pm 0.005^{\mathrm{a}^{\prime}}$ & $0.001 \pm 0.003^{\mathrm{b}^{\prime}}$ & $0.002 \pm 0.002^{c^{\prime}}$ & $0.005 \pm 0.008^{\mathrm{d}^{\prime}}$ \\
\hline HCC stage: I/II/III & $10 / 14 / 2$ & $7 / 15 / 0$ & $0 / 3 / 0$ & $4 / 5 / 0$ \\
\hline \multicolumn{5}{|l|}{ AJCC } \\
\hline pT1N0 & $10(38.5)$ & $7(31.8)$ & 0 & $4(44.4)$ \\
\hline pT2N0 & $14(53.8)$ & $15(68.2)$ & $3(100)$ & $5(55.6)$ \\
\hline pT3N0 & $2(7.7)$ & 0 & 0 & 0 \\
\hline \multicolumn{5}{|l|}{ Knodell score } \\
\hline $\mathrm{F} 2$ & 0 & $1(4.5)$ & 0 & $1(11.1)$ \\
\hline F3 & $1(3.8)$ & 0 & 0 & 0 \\
\hline $\mathrm{F} 4$ & $25(96.2)$ & $21(95.5)$ & $3(100)$ & $8(88.9)$ \\
\hline \multicolumn{5}{|l|}{ Fatty liver } \\
\hline Negative & $12(46.1)$ & $9(41.0))$ & $1(33.3)$ & $5(55.6)$ \\
\hline$<5 \%$ & $9(34.6)$ & $11(50.0)$ & $1(33.3)$ & $4(44.4)$ \\
\hline $6-10 \%$ & $4(15.4)$ & $1(4.5)$ & 0 & 0 \\
\hline$>15 \%$ & $1(3.9)$ & $1(4.5)$ & $1(33.3)$ & 0 \\
\hline
\end{tabular}

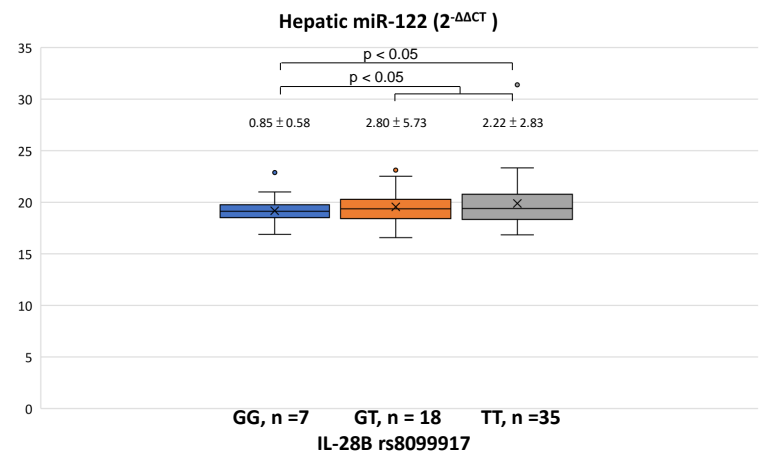

Fig. 3 Hepatic miR-122 expression was significantly higher in patients with the genotype TT than in those with genotype GG $(p<0.05)$, and higher hepatic miR122 expression was observed in those with genotype TT plus genotype GT compared to in those with genotype GG $(p<0.05)$.

and $>6 \%$ and without fatty plus $<5 \%$ fatty liver groups $(p<0.005)$ (Fig. 4).

All patients adhered to BCLC treatment guidelines and had low serum alpha fetoprotein (AFP) levels before LDLT. The median follow-up time of the patients was 20.5 (10-23) months. In this period, no patients showed evidence of HCC recurrence or viral hepatitis relapse after LDLT.

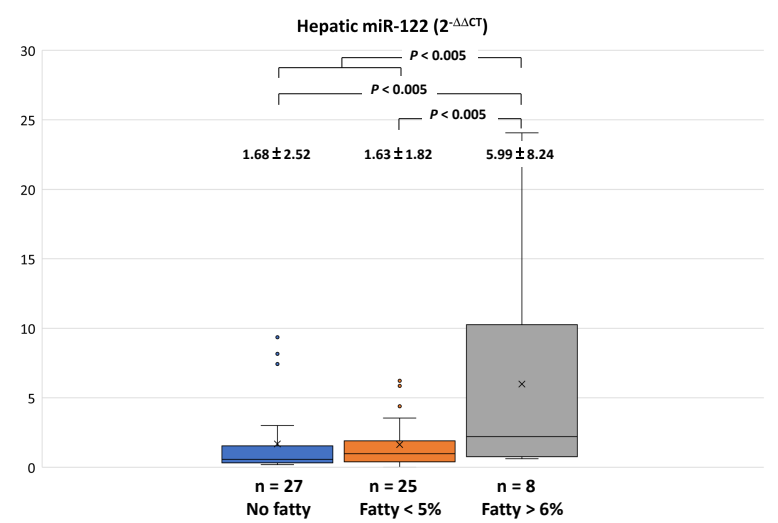

Fig. 4 Hepatic miR-122 expression was significantly different between the native liver with $>6 \%$ fatty liver and without fatty liver $(p<0.005)$ as well as between $>6 \%$ fatty liver and $<5 \%$ fatty liver $(p<0.005)$ and between $>6 \%$ fatty and without fatty plus $<5 \%$ fatty liver $(p<0.005)$.

\section{Hepatic miR-122 and IL-28B polymorphism associated with the outcomes of the HCC patients after liver transplantation}

In 2-year follow-up observation, none of the patients developed HBV or HCV recurrence after LDLT. Although full fit the BCLC transplantation criteria, 
$1.67 \%(1 / 60)$ of patients with HCC recurrence presented with distant metastasis, of which high expression of hepatic miR-122 and IL-28B rs8099917 genotype TT associated with major vessel invasion in the explanted liver.

\section{DISCUSSION}

According to our results, miR-122 expression showed large increases in the liver tissue and very low increases in the plasma in LDLT patients, which agrees with a previous study in adult male rats [9]. Particularly hepatic miR-122 was detected in the native liver following LDLT. Therefore, hepatic miR122 is a reliable genetic marker. In addition to viral factors, host characteristics have important effects on HCC recurrence. This is the first study to explore both hepatic miR-122 and IL-28B genetic polymorphisms in patients with HCC. In the current study, $100 \%$ of serum samples were positive for HBsAg and $72.4 \%(21 / 29)$ for HBsAg in hepatocytes from the native liver. Complete HBsAg seroconversion to anti-HBs can be achieved using optimal treatment strategies before and after LDLT. Single-nucleotide polymorphisms in miR-122 were previously suggested to be associated with HCC [10]; however, hepatic miR-122 expression did not significantly differ between patients with different etiologies as $\mathrm{HBV}$, HCV, dual HBV/HCV, and non-B non-C in this study.

The IL-28B rs8099917 polymorphism genotypes TT and GT are associated with a positive response to chronic $C$ hepatitis treatment [11]. In this study, hepatic miR-122 expression was correlated with the rs8099917 genotypes TT and GT, but not with the rs12979860 polymorphism. Therefore, hepatic miR-122 and IL-28B genetic polymorphisms may indicate benefit outcomes in patients with HCV and in those with HBV infection. Our patients had chronical viral hepatitis complications with liver cirrhosis and HCC after liver transplantation, and 95\% showed F4 fibrosis according to pathological investigation. High miR-122 expression was previously reported to be correlated with liver fibrosis staging [12].

At the post-transcriptional level, miR-122 regulates multiple physiological and pathological functions in both alcoholic and non-alcoholic fatty liver diseases through modulating gene expression [13]. In our pathological investigation, hepatic miR-122 was highly correlated with $>6 \%$ fatty liver in the native liver following LDLT. In contrast, lower hepatic miR-122 expression was detected in cases without fatty liver and $<5 \%$ non-alcoholic fatty liver. Fur- thermore, miR-122 has been reported as a potential diagnostic marker for distinguishing patients with non-alcoholic fatty liver disease from healthy subjects. Upregulated miR-122 is associated with the severity of liver fibrosis, with deregulation observed at different stages of fatty liver disease $[14,15]$, as observed in the present study where miR-122 was significantly correlated with the clinicopathologics particularly in hepatic fibrosis and fatty liver component. In the contrary, hepatic miR-122 expression was correlated with the rs8099917 genotypes TT and GT but not with the rs12979860 polymorphism in the clinicopathological presentation. Because the miR-122 expression showed large increases in the liver tissue and very low increases in the plasma in LDLT patients, there was no significant correlation between the hepatic and plasma miR-122s in all patients or in the subgroups. Limitations of this study included the small number of cases, the short duration of evaluation, the lack of a healthy control group, as all patients underwent LDLT.

In conclusion, miR-122 expression was significantly higher in the native liver tissue than in the plasma. The high hepatic levels of miR-122 were correlated with the IL-28B rs8099917 genotypes TT and GT and with $>6 \%$ fatty liver, and thus may play a major role in HCC.

Acknowledgements: We thank Ms. Ching-Yin Huang for her assistance with data collection. This work was supported by grant number CMRPG8F1541 from the Chang Gung Memorial Hospital of Taiwan.

\section{Abbreviation}

AFP, alpha fetoprotein; anti-HBe, antibody for hepatitis B e; anti-HBs, antibody for hepatitis B surface; ant-HCV, antibody for hepatitis $C$ virus; BCLC, Barcelona Clinic Liver Cancer; DNA, deoxyribonucleic acid; $\mathrm{HBcAg}$, hepaitis $\mathrm{B}$ core antigen; HBeAg, hepatitis B e antigen; HBsAg, hepatitis B surface antigen; HBV, hepatitis B virus; HCC, hepatocellular carcinoma; HCV, hepatitis C virus; LDLT, living donor liver transplantation; miRNA, microRNA; RNA, ribonucleic acid; and RT-PCR, reverse transcription-polymerase chain reaction.

\section{REFERENCES}

1. Skalsky RL, Cullen BR (2010) Viruses, microRNAs, and host interactions. Annu Rev Microbiol 64, 123-141.

2. Qiao DD, Yang J, Lei XF, Mi GL, Li SL, Li K, Xu CQ, Yang HL (2017) Expression of microRNA-122 and microRNA-22 in HBV-related liver cancer and 
the correlation with clinical features. Eur Rev Med Pharmacol Sci 21, 742-747.

3. Zhang Y, Li Y, Jiang W, Li Q, Lan Y (2019) The clinical significance of microRNA-122 in predicting the prognosis of patients with hepatocellular carcinoma: a meta-analysis validated by the Cancer Genome Atlas dataset. Medicine (Baltimore) 98, e14810.

4. Wen Y, Han J, Chen J, Dong J, Xia Y, Liu J, Jiang Y, Dai $\mathrm{J}$, et al (2015) Plasma miRNAs as early biomarkers for detecting hepatocellular carcinoma. Int J Cancer 137, 1679-1690.

5. Chiu KW, Nakano T, Chen KD, Lin CC, Hu TH, Goto S, Chen CL (2016) Association of IL28B SNPs rs12979860 and rs8099917 on hepatitis C virusRNA status in donors/patients of living donor liver transplantation. PLoS One 11, e0156846.

6. Su TH, Liu CH, Liu CJ, Chen CL, Ting TT, Tseng TC, Chen PJ, Kao JH, et al (2013) Serum microRNA-122 level correlates with virologic responses to pegylated interferon therapy in chronic hepatitis C. Proc Natl Acad Sci USA 110, 7844-7849.

7. Nakano T, Chen IH, Goto S, Lai CY, Tseng HP, Hsu LW, Chiu KW, Lin CC, et al (2017) Hepatic miR-301a as a Liver transplant rejection biomarker? and its role for interleukin-6 production in hepatocytes. OMICS 21, 55-66.

8. Yen YH, Huang CM, Wei KL, Wang JH, Lu SN, Lee CM, Hung CH, Chen CH, et al (2016) MicroRNA-122 as a predictor of HBsAg seroclearance in hepatitis $\mathrm{B}$ and $C$ dual infected patients treated with interferon and ribavirin. Sci Rep 6, ID 33816.

9. Camacho L, Silva CS, Hanig JP, Schleimer RP, George NI, Bowyer JF (2019) Identification of whole blood mRNA and microRNA biomarkers of tissue damage and immune function resulting from amphetamine exposure or heat stroke in adult male rats. PLoS One 14, e0210273.

10. Bei C, Liu S, Yu X, Qiu M, Tang B, Liao W, He S, Yu $\mathrm{H}$ (2018) Single nucleotide polymorphisms in miR122 are associated with the risk of hepatocellular carcinoma in a southern Chinese population. Biomed Res Int 2018, ID 1540201.

11. Cheng $\mathrm{CH}$, Lin CC, Chen HL, Lin IT, Wu CH, Lee YK, Wong MW, Bair M (2017) High frequencies of a favorable IL-28B rs8099917 polymorphism and the clinical implications in patients with $\mathrm{HCV}$ in one multiracial area of Taiwan. Kaohsiung J Med Sci 33, 510-515.

12. Wang TZ, Lin DD, Jin BX, Sun XY, Li N (2019) Plasma microRNA: A novel non-invasive biomarker for HBVassociated liver fibrosis staging. Exp Ther Med 17, 1919-1929.

13. Torres JL, Novo-Veleiro I, Manzanedo L, AlvelaSuárez L, Macías R, Laso FJ, Marcos M (2018) Role of microRNAs in alcohol-induced liver disorders and non-alcoholic fatty liver disease. World $J$ Gastroenterol 24, 4104-4118.

14. Liu CH, Ampuero J, Gil-Gómez A, Montero-Vallejo R, Rojas Á, Muñoz-Hernández R, Gallego-Durán R, Romero-Gómez M (2018) miRNAs in patients with non-alcoholic fatty liver disease: A systematic review and meta-analysis. J Hepatol 69, 1335-1348.

15. Wang Q, Ma J, Jiang Z, Wu F, Ping J, Ming L (2017) Identification of microRNAs as diagnostic biomarkers for acute myocardial infarction in Asian populations: A systematic review and meta-analysis. Medicine (Baltimore) 96, e7173. 